Article

\title{
Feasibility of Using Graphene Oxide Nanoflake (GONF) as Additive of Cement Composite
}

\author{
Jinwoo An ${ }^{1}$, Matthew McInnis ${ }^{2}$, Wonseok Chung ${ }^{3, *}$ and Boo Hyun Nam ${ }^{1, *}$ \\ 1 Department of Civil, Environmental, and Construction Engineering, University of Central Florida, \\ 4000 Central Florida Blvd, Orlando, FL 32816, USA; jinwooan@knights.ucf.edu \\ 2 Manager of Applied Technology, Garmor Inc. 6317 McCoy Road. Suite 100, Orlando, FL 32822, USA; \\ mmcinnis@garmortech.com \\ 3 Department of Civil Engineering, Kyung Hee University, 1732 Deogyeong-daero, Giheung-gu, Yongin-si, \\ Gyeonggi-do 17104, Korea \\ * Correspondence: wschung@khu.ac.kr (W.C.), boohyun.nam@ucf.edu (B.H.N.)
}

Received: 17 November 2017; Accepted: 11 February 2018; Published: 12 March 2018

Featured Application: Admixture (additive) for cement composites.

\begin{abstract}
The object of this study is to investigate the feasibility of ball-milled graphene oxide nanoflakes (GONFs) produced by a mechanochemical process as an additive in Ordinary Portland Cement (OPC)-based paste and concrete. Different percentages of GONFs (0.01-1 wt. \%) were added in OPC paste mix to find the optimum content of GONF in GONF-combined cement composites. To investigate the effect of the changes in the mix design on OPC paste, two mix design methods were employed: (1) Dry-mix, where GONFs and OPC powders are mixed prior to paste formation and (2) Wet-mix, where sonicated GONFs solution is used as the pour water with OPC paste mix. Data from mechanical tests (compressive and flexural tests) showed that OPC paste incorporating $0.05 \%$ GONF resulted in the highest strength for OPC paste. The results of strength tests indicated that Dry-mix is competitive as compared with Wet-mix. Scanning electron microscope (SEM) and energy dispersive spectroscopy (EDS) was used to identify GONFs inside products of cement hydration. To explore the practicality of using GONF in concrete, concrete specimens were made, cured and tested with different percentages of dry-mixed GONFs (0.01-0.1 wt. \%). The result of strength test showed that GONF improves the compressive and flexural strengths of concrete around $15 \%$ with 0.01 wt. \% GONF.
\end{abstract}

Keywords: ball-milled graphene oxide; cement hydrates; petrographic analysis; dry-mix design

\section{Introduction}

In recent decades, carbon-based nanoscale materials such as graphene, carbon nanofiber (CNF), graphene oxide nanoflake (GONF), and carbon nanotubes (CNT) have been studied and applied as reinforcing materials of cement-based mixtures, because of their unique combination of properties (high tensile strength and modulus, surface area and electrical conductivity). Many researchers reported that carbon-based nanoscale materials improve the mechanical properties of Ordinary Portland Cement (OPC) composites [1-3]. Nano-reinforcements in cementitious matrix materials differ from conventional reinforcements at the millimeter scale and larger because they can control nano-size cracks before they develop into micro- and meso-size cracks [4-8].

GONF, in particular, has received attention from civil engineering researchers among carbon-based nanoscale materials due to the oxygen groups on the surface of GONFs. These oxygen groups are thought to coordinate ions to initiate the nucleation of cement hydrates, such as calcium silicate hydrate (C-S-H), calcium hydroxide $\left(\mathrm{Ca}(\mathrm{OH})_{2}\right), \mathrm{AFt}$ (ettringite) and AFm 
(monosulphate), etc. $[2,3,6,9,10]$, thereby making GONF a seeding material. Moreover, GONF's nano-filling and nano-bridging mechanism in the cement matrix has been introduced by other researchers [3,9,11,12]. Mohammed et al. [5] reported that cement composite with the addition of very low fraction of graphene oxide (GO) $(0.01 \%)$ can hinder the penetration of chloride ions. Tong et al. [13] discovered that incorporation of GO in cementitious materials improves not only the strength but also corrosion resistance and freeze-and-thaw performance. Lu et al. [6] found that the incorporation of $0.08 \%$ GO in Strain Hardening Cementitious Composites (SHCCs) increases $24.8 \%$ of compressive strength and $37.7 \%$ of flexural strength. Moreover, flexural toughness was increased up to $105 \%$. Gong et al. revealed that the introduction of $0.03 \%$ of GONF into cement paste leads to increment of compressive and flexural strengths of GO-cement composite by more than $40 \%$, because of the reduction of porosity of cement paste [12]. In addition, it was mentioned that the total amount of nonevaporable water and calcium hydroxide in cement paste was increased. Furthermore, other researchers also reported that the incorporation of GO improves the mechanical properties and microstructure of cement-based composite $[3,9,11,14]$.

GONFs used in previous studies have been made using Hummer's method [2,3,5-9,11,12], a chemical process of oxidizing graphite, which is untenable as an additive in cement and concrete because of cost and scalability. Traditional methods for producing GONFs, such as Hummer's method, depend on strong and somewhat harmful oxidizing agents and acids $\left(\mathrm{H}_{2} \mathrm{O}_{2}, \mathrm{H}_{2} \mathrm{SO}_{4}\right.$ and $\left.\mathrm{KMnO}_{4}\right)$ and produces significant quantities of acidic byproduct [1]. Figure 1 shows the typical manufacturing process of GONFs through the chemical process.

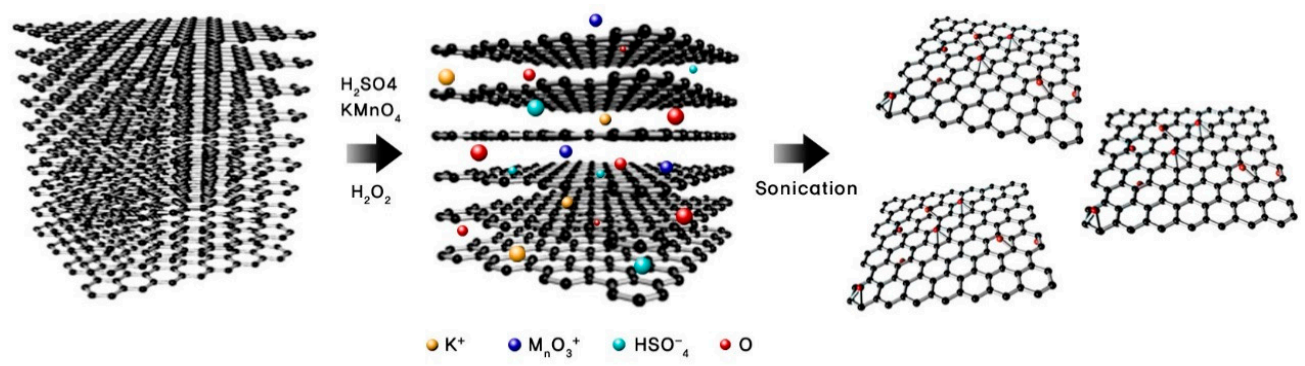

Figure 1. A proposed chemical reaction pathway for producing graphene oxide (reproduced with permission from [15]. Copyright Elsevier, 2016).

GONFs used in this study were produced using an innovative mechanochemical process which uses milling technology like those in the mining industry with common reactants yielding GONFs more cost-effectively and scalably (see Figure 2). This innovative technology directly mills graphite power with a non-toxic oxidizing agent using conditions that minimize collision forces and optimize shearing forces thus graphite powder is simultaneously oxidized and delaminated with a few layers suitable for the manifold purpose. These proprietary achievements eliminate hazardous waste disposal costs and deliver a product suitable for large-scale production at commodity-type prices [16,17]. This promising alternative method to produce large-scale production of GONFs is a requirement for introducing GONFs into the concrete industry. One of the major hindrances encountered by previous researches which prevented the introduction of GONFs into concrete (relatively large scale) is the small production of GONFs. This major hindrance can be overcome by addressing this innovative technology. Figure 2 shows the manufacturing process of ball-milling GONF.

The other hindrance to introduce GONFs into concrete is the dispersion issue of GONFs in concrete mix. It is well known that the best method for dispersing of GONFs in a solvent such as water is by applying sonication [1,11]. All previous researchers have used GONF-solution to make GONF-combined cement composites $[3,5,6,9,12]$. Originality, the uniqueness of this study, is to disperse GONFs as powder in OPC prior to mixing with water to investigate the feasibility of using GONFs as dry-addition. Some of previous studies have used either surfactant or dispersant with water to 
improve the dispersion of GONFs [18-20]. However, none of the previous studies used dry GONF powder for the cement composite mix without applying sonication.

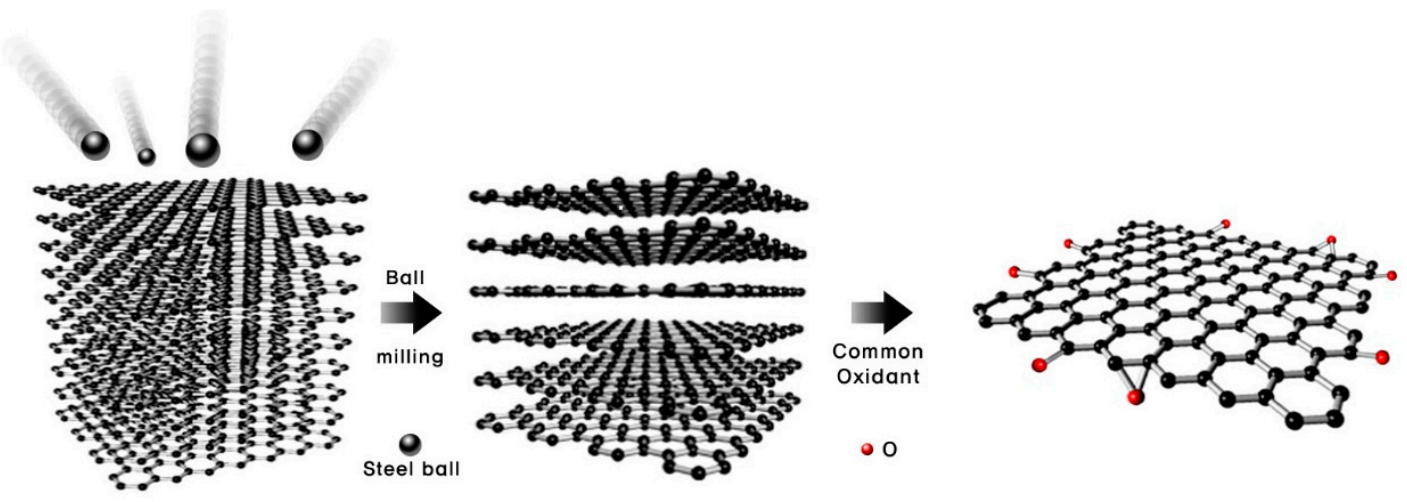

Figure 2. Mechanochemical process of producing ball-milling graphene oxide nanoflake (reproduced with permission from [15]. Copyright Elsevier, 2016).

In general, concrete mix requires relatively large amounts of water when compared with cement paste mix due to larger scale specimens and structure. Thus, for the design of GONF-combined cement paste mix (relatively small scale), most of the previous studies used GONFs dispersed in water by applying sonication. Figure 3 shows dry GONF powder and GONF dispersed solution. For concrete mix, however, it requires a relatively large quantity of water. Therefore, applying sonication for the dispersion of GONFs into water can be a major issue. The construction industry typically uses ready mixed concrete. Each batch of ready mixed concrete is tailor-made and normally consumes a high volume of water. Even though the sonication method is an effective method to disperse GONFs in water, it has some imitations, especially for high volume process. One of limitations is the difficulty of reproducibility of sonication results. The transmitted energy is highly depending on the location of the probe. In other words, the sonicator has a limited range of transmitted energy around the probe. In this study, two different mix designs were employed to investigate the effect of mix design method on the mechanical properties of OPC based specimens. The applied two mix designs are called Dryand Wet-mix designs. Dry-mix design indicates the GONF is mixed with cement as a dry power while Wet-mix design signifies that the GONF as a solution (after sonication of GONF with water) is mixed with cement. Dry-mix is more practical and effective for concrete mix due to the ease of preparation, mixing and placing of concrete mix. Wet-mix is useful as a more direct comparison to published literature as a baseline for GONF efficacy in cement and concrete.

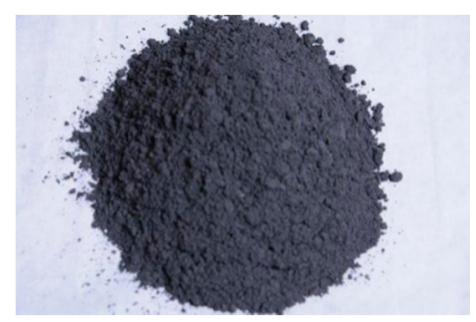

(a)

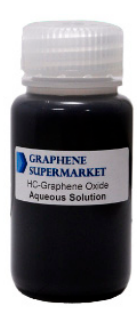

(b)
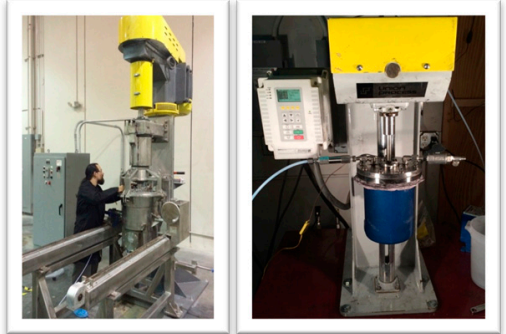

(c)

Figure 3. Graphene oxide nanoflake (GONF): (a) Dry GONF powder; (b) GONF-solution; and (c) the equipment for the ball milling process [16]. 


\section{Materials and Mix Designs}

\subsection{Materials}

Ordinary Portland Cement (OPC) Type-I conforming to American Society for Testing and Materials (ASTM) C 150 [21] was used in both the cement paste and concrete as the primary binding material for this research. Table 1 shows the chemical composition of OPC. As shown in the table, major elements are calcium and silicon.

Table 1. Chemical composition of Ordinary Portland Cement.

\begin{tabular}{cccccccc}
\hline Contents & $\mathrm{CaO}$ & $\mathrm{SiO}_{2}$ & $\mathrm{SO}_{3}$ & $\mathrm{Al}_{2} \mathbf{O}_{3}$ & $\mathrm{Fe}_{2} \mathbf{O}_{3}$ & Insoluble Residue & Total Loss on Ignition \\
\hline Test value (\%) & 64.90 & 21.49 & 0.70 & 4.21 & 3.50 & 1.10 & - \\
\hline
\end{tabular}

On the contrast, the major chemical element of GONF is carbon (see Table 2). As can be seen in Table 2, more than $99 \%$ of the non-oxygen chemical composition of GONFs is carbon. The GONF was supplied by Garmor, Inc. (Orlando, FL, USA) [16] and the GONF products are achieved by milling graphite powder with an oxidizing agent and under controlled conditions with optimum shearing forces and minimized collision forces. This results in oxidizing graphite along the edges while the milling media simultaneously exfoliates the layers in a zipper-like fashion. The process yields edge-oxidized graphene oxide composed of a few layers of graphene. This mild oxidation process preserves the carbon $\mathrm{sp}^{2}$ orbitals on the surface of the flake, which enhances electrical conductivity and other properties as well. The GONF is hydrophilic, readily suspends in water, and can be further chemically functionalized.

Table 2. Chemical Composition of GONFs (edge-oxidized) [16].

\begin{tabular}{cccccccc}
\hline \multirow{2}{*}{ Oxygen } & \multicolumn{7}{c}{ Non-Oxygen Composition } \\
\cline { 2 - 8 } & Carbon & Silicon & Sulphur & Potassium & Calcium & Chromium & Copper \\
\hline $5 \sim 10 \%$ & $>99.8 \%$ & $<40 \mathrm{ppm}$ & $<60 \mathrm{ppm}$ & $<5 \mathrm{ppm}$ & $<30 \mathrm{ppm}$ & $<125 \mathrm{ppm}$ & $<5 \mathrm{ppm}$ \\
\hline
\end{tabular}

For concrete mix, Florida Department of Transportation (FDOT) certified sand passing $4.75 \mathrm{~mm}$ was obtained from CEMEX and used as a fine aggregate. The gradation curve of the sand met the ASTM requirement (ASTM C33 [22]) as shown in Figure 4. Fineness modulus of the fine aggregate was 2.36, which also complies with the American Concrete Institute (ACI) requirement (2.3 to 3.1). Limestone obtained from CEMEX with nominal maximum size of $19 \mathrm{~mm}$ was used as a coarse aggregate. The sieve analysis for the coarse aggregate (Figure 5) demonstrated that the particle sizes for the coarse aggregate fell inside the ASTM certified zone (ASTM C 136 [23]).

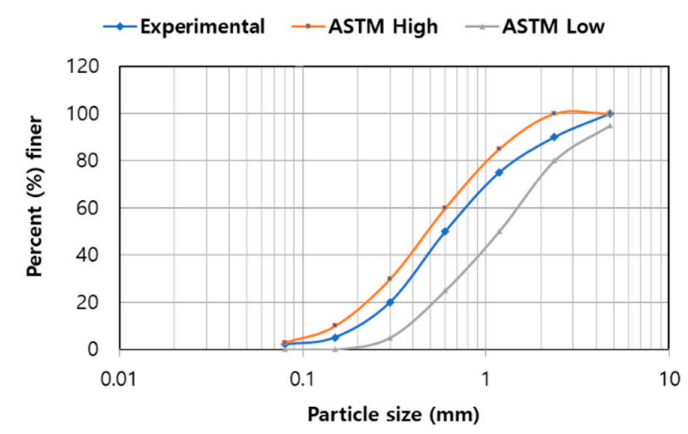

Figure 4. Gradation curves for fine aggregate and American Society for Testing and Materials (ASTM) C33 grading requirements for fine aggregate. 


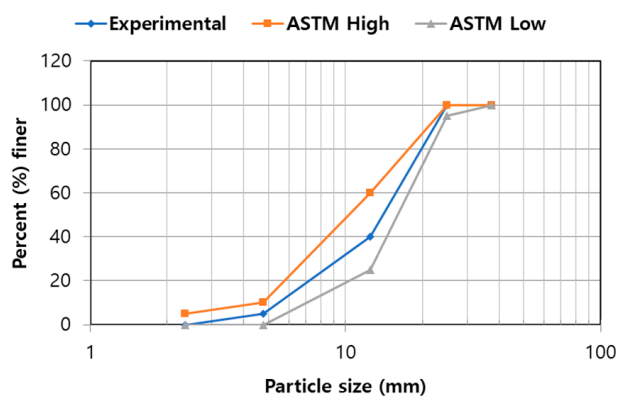

Figure 5. Gradation curves for coarse aggregates and ASTM C33 grading requirements for No. 57 aggregate.

\subsection{Mix Design}

The GONF-cement paste was prepared by mixing OPC and water with the different percentages of GONFs (0.01-1 wt. \%). For the comparison of different types of mix design, two different mix design methods were employed (Dry-mix and Wet-mix designs). Dry-mix design represents that the GONF powder is mixed with cement grains in a shear mixer prior to mixing with water. Wet-mix design represents that GONF powder is dissolved into water by sonication, and the GONF solution is then mixed with cement. For the dispersion of GONF powder in OPC powder (Dry-mix design), (1) one kilogram of OPC power is placed in a zip-lock plastic bag; (2) GONF power is spread on OPC and the bag is sealed; (3) The zip-lock plastic bag is rubbed with hands for a minute to apply shearing force and (4) one kilogram of GONF-mixed OPC is poured in the rest of OPC power for the mix and mixed with a portable mixer for a minute. For the dispersion of Wet-mix design, (1) the required amount of GONF powder is poured into one gallon of water; (2) the sonicator is placed in GONF-mixed water; and (3) the sonicator is operated for a minute prior to the mixing. The GONF was used as an additive in cement paste and the GONF content was varied from $0.1 \%$ to $1 \%$ with respect to the weight of OPC in the mix. For each mix design, three specimens were prepared for mechanical tests after curing. A weight ratio of water to cement of 50:100 (w/c 0.5) is used for the GONF-cement composite. Cubic specimens of GONF-cement composites (dimension of $50 \mathrm{~mm} \times 50 \mathrm{~mm} \times 50 \mathrm{~mm}$ ) were prepared for compressive strength testing. The sample dimensions for flexural strength testing of cement paste was $50 \mathrm{~mm} \times 100 \mathrm{~mm} \times 25 \mathrm{~mm}$. The samples were cured at $20{ }^{\circ} \mathrm{C}$ and $90 \%$ relative humidity until testing. Detailed mix proportioning of control and GONF-cement pastes are shown in Table 3. The specimen ID is denoted as P (cement paste), PD (GONF-combined paste with Dry-mix design) and PW (GONF-combined paste with Wet-mix design).

For GONF-concrete, OPC, water, fine and coarse aggregates were mixed with different percentages of GONFs (0.01-0.1 wt. \%). Detailed mix proportioning of concrete mix can be found from Table 3 . Unlike GONF-combined paste mix (0.01-0.1 wt. \% of GONF), GONF was added to concrete mix from $0.01 \%$ to $0.1 \mathrm{wt}$. \% since the optimum content of GONF was found as $0.05 \%$ for the improvement of GONF-combined paste. Moreover, only Dry-mix design was used for the concrete mix. To make GONF solution for Wet-mix, applying sonication is necessary. However, applying sonication to a large amount of water is ineffective as well as not practical for the concrete industry. Therefore, Wet-mix design may not be feasible for field construction. The specimen ID is referred as C (concrete) and CD (GONF-combined concrete with Dry-mix design).

Table 3. Mix proportions of GONF-cement paste and concrete specimens $\left(\mathrm{kg} / \mathrm{m}^{3}\right)$.

\begin{tabular}{ccccccc}
\hline \multirow{2}{*}{ Specimen ID } & w/c & Water & Cement & GONF & Fine Agg. & Coarse Agg. \\
\cline { 2 - 7 } & Ratio & $\left(\mathbf{k g} / \mathbf{m}^{\mathbf{3}}\right)$ & $\mathbf{( k g / \mathbf { m } ^ { 3 } )}$ & $\mathbf{( k g / \mathbf { m } ^ { 3 } )}$ & $\mathbf{( k g / \mathbf { m } ^ { 3 } )}$ & $\mathbf{( k g / \mathbf { m } ^ { 3 } )}$ \\
\hline $\mathrm{P}$ & 0.5 & 180 & 360 & - & - & - \\
PD0.01 & 0.5 & 180 & 360 & 0.036 & - & - \\
\hline
\end{tabular}


Table 3. Cont.

\begin{tabular}{ccccccc}
\hline \multirow{2}{*}{ Specimen ID } & w/c & Water & Cement & GONF & Fine Agg. & Coarse Agg. \\
\cline { 2 - 6 } & Ratio & $\mathbf{( k g / \mathbf { m } ^ { \mathbf { 3 } } )}$ & $\mathbf{( k g / \mathbf { m } ^ { \mathbf { 3 } } )}$ & $\mathbf{( k g / \mathbf { m } ^ { \mathbf { 3 } } )}$ & $\mathbf{( \mathbf { k g } / \mathbf { m } ^ { \mathbf { 3 } } \mathbf { ) }}$ & $\mathbf{( k g / \mathbf { m } ^ { \mathbf { 3 } } )}$ \\
\hline PD0.05 & 0.5 & 180 & 360 & 0.18 & - & - \\
PD0.1 & 0.5 & 180 & 360 & 0.36 & - & - \\
PD0.5 & 0.5 & 180 & 360 & 0.36 & - & - \\
PD1 & 0.5 & 180 & 360 & 3.6 & & - \\
PW0.01 & 0.5 & 180 & 360 & 0.036 & - & - \\
PW0.05 & 0.5 & 180 & 360 & 0.18 & - & - \\
PW0.1 & 0.5 & 180 & 360 & 0.36 & - & - \\
PW0.5 & 0.5 & 180 & 360 & 0.36 & - & \\
PW1 & 0.5 & 180 & 360 & 3.6 & & \\
C & 0.5 & 180 & 360 & - & 761 & 949 \\
CD0.01 & 0.5 & 180 & 360 & 0.036 & 761 & 949 \\
CD0.02 & 0.5 & 180 & 360 & 0.072 & 761 & 949 \\
CD0.03 & 0.5 & 180 & 360 & 0.108 & 761 & 949 \\
CD0.04 & 0.5 & 180 & 360 & 0.144 & 761 & 949 \\
CD0.05 & 0.5 & 180 & 360 & 0.18 & 761 & 949 \\
CD0.1 & 0.5 & 180 & 360 & 0.36 & 761 & 949 \\
\hline
\end{tabular}

\section{Experimental Methods}

To characterize the properties of GONF, atomic force microscopy (AFM) and laser diffraction, scanning electron microscopy (SEM) were used. The mini-slump test [24] and setting time test [25] were used to investigate the properties of freshly mixed GONF-combined paste. Both compressive strength test (ASTM C109) and flexural strength test (ASTM C348) were conducted on the hardened GONF-combined pastes and concretes to evaluate the effect of GONF content and different mix designs on strength. Three specimens were prepared for each mix design; thus, the strength tests were conducted three times for each mix design and the results were averaged. In addition, the microstructure of the hardened GONF-cement composites were investigated using scanning electron microscope (SEM) and energy dispersive spectroscopy (EDS). SEM and EDS were performed to investigate surface morphology of GONF, microstructure and chemical composition of cement hydration products when GONF is introduced into cement hydration.

\section{Results and Discussion}

\subsection{Characterization of GONF}

Carbon based nanomaterials are unique with respect to their superior properties, which include, but not limited to, high tensile strength and elastic modulus, high surface area and electrical conductivity [1,11]. Mechanical and physical properties of the GONF used in this study were characterized with atomic force microscopy (AFM) and BET sorbtometery. Table 4 shows the comparison of OPC and GONF. It is known that mechanical properties (elastic modulus and tensile strength) of GONF are higher than steel [1,11]. Moreover, GONF is an ultra-fine material according to surface area in Table 4.

Table 4. Basic physical properties of materials, standard test methods and devise.

\begin{tabular}{cccc}
\hline Properties & OPC & GONF & Test Methods and Device \\
\hline Elastic modulus & - & $1 \mathrm{TPa}$ & Atomic Force Microscope (AFM) \\
Tensile Strength & - & $130 \mathrm{GPa}$ & AFM \\
Specific gravity (OD) & 3.15 & 1.91 & ASTM C127 [26], C128 [27] \\
Surface area & $33.10 \mathrm{~m}^{2} / \mathrm{g}$ & $204 \mathrm{~m}^{2} / \mathrm{g}$ & BET Sorptometery \\
\hline
\end{tabular}

In addition, carbon based nanomaterials have the diversity in their morphologies from oneto three-dimension. The surface morphology of GONF was investigated with scanning electron 
microscopy (SEM). SEM image of GONF which used in this study is presented in Figure 6 (see Figure 6c [16]) as compared with other carbon-based nanomaterials such as carbon nanofiber (CNF, Figure 6a [28]), graphene (Figure 6b [29]) and carbon nanotube (CNT, Figure 6d [30]). As can be seen in Figure 6, CNF, graphene and CNT appear in one-, two- and three- dimension morphologies. Each material has its own merit with their morphologies. As shown in Figure 6c, GONF is a wrinkled layer due to intercalated functional oxygen groups on the surface of graphene (see Table 2). It is found that a nominal particle size diameter of GONF is $500 \mathrm{~nm}$ with $90 \%$ of the particle sizes below $800 \mathrm{~nm}$ in diameter. The particle size of GONF can be tailored based on the starting material which is graphite and the mechanochemical processing conditions.

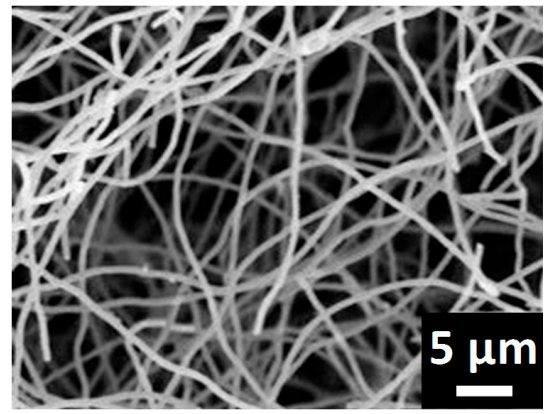

(a)

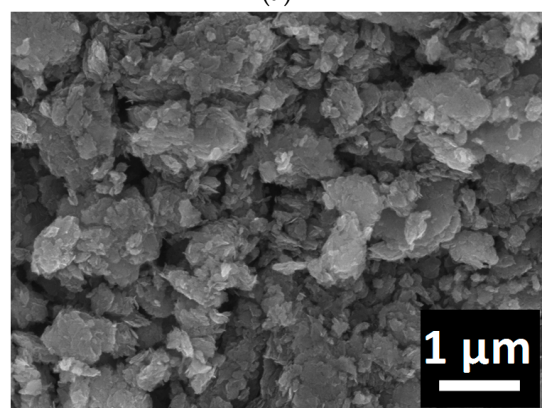

(c)

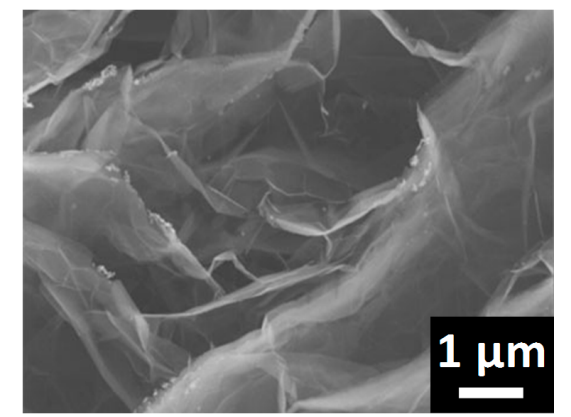

(b)

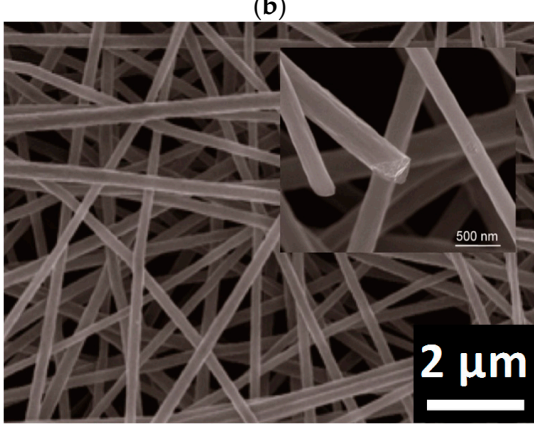

(d)

Figure 6. SEM images of various carbon-based materials ((a) carbon nanofiber (CNF); (b) grapheme; and (d) carbon nanotubes (CNT) and (c) GONF which used in this study. (conversion: $1 \mu \mathrm{m}=0.0394 \mathrm{mil}$ ) (reproduced with permission from [28]. Copyright IOP Publishing, 2014).

\subsection{Effect of GONF on the Properties of Fresh Cement Paste}

The effect of GONF on the properties of freshly mixed cement paste was investigated by performing the mini-slump test and measuring the setting time. The mini-slump flow test was employed to determine the influence of GONF on the workability of cement paste. For the mini-slump test, a $600 \mathrm{~g}$ of OPC was used with $0.5 \mathrm{w} / \mathrm{c}$ ratio. OPC and water was mixed in a mixer for $1 \mathrm{~min}$. Then the mixture was poured into a mini slump cone, compacted, struck off and lifted at $2 \mathrm{~min}$. The area of each pat was determined and reported. Table 5 shows the results of mini-slump tests of OPC paste with different percentages of GONF. The diameter of the mini-slump flow test is approximately $110 \mathrm{~mm}$. The addition of GONF in OPC paste results in the decrement of mini-slump flow. With the increment of the dosages of GONF, the diameter of mini-slump flow was decreased proportionally. This result indicates that the addition of GONF reduces the fluidity of OPC paste mix. This phenomenon might be explained with the physical and chemical properties of GONF. GONF has a large surface area compared to OPC (see Table 4). Therefore, GONF could absorb and hold more water molecules around it during mixing. The other reason of the reduction of fluidity can be due to oxygen groups at the edge of GONF. These oxygen groups may initiate the cement hydration process faster by reacting with ions from the dissolved cement grains $[9,13]$. 
Table 5. The results of mini slump tests, initial and final setting times.

\begin{tabular}{cccc}
\hline Specimen ID & Mini Slump Test $\mathbf{( c m}^{2}$ ) & Initial Setting (hour) & Final Setting (hour) \\
\hline P & $155.48(100 \%)$ & $5.75(100 \%)$ & $9.5(100 \%)$ \\
PD0.01 & $155.32(99.9 \%)$ & $5.55(96.5 \%)$ & $9.25(97.4 \%)$ \\
PD0.05 & $121.28(78 \%)$ & $5.3(92.2 \%)$ & $9.1(95.8 \%)$ \\
PD0.1 & $116.61(75 \%)$ & $5.5(95.7 \%)$ & $9.45(99.5 \%)$ \\
PD0.5 & $107.28(69 \%)$ & $5.85(101.7 \%)$ & $10(105.3 \%)$ \\
PD1 & $93.29(60 \%)$ & $6.05(105.2 \%)$ & $10.1(106.3 \%)$ \\
PW0.01 & $155.42(99.9 \%)$ & $5.75(100 \%)$ & $9.65(101.6 \%)$ \\
PW0.05 & $129.05(83 \%)$ & $5.5(95.7 \%)$ & $9.5(100 \%)$ \\
PW0.1 & $122.83(79 \%)$ & $5.8(100.9 \%)$ & $9.75(102.6 \%)$ \\
PW0.5 & $111.95(72 \%)$ & $5.9(102.6 \%)$ & $10.1(106.3 \%)$ \\
PW1 & $105.73(68 \%)$ & $6.15(107 \%)$ & $10.55(111.1 \%)$ \\
\hline
\end{tabular}

One of the indirect methods to determine the process of cement hydration is the time of setting. The strength of cementious materials is gained with the growth of cement hydration products. The time of initial setting can be measured when OPC paste begins to stiffen and the time of final set can be found when the cement has hardened to sustain some load. For the setting time test, a $400 \mathrm{~g}$ of OPC was used with $0.5 \mathrm{w} / \mathrm{c}$ ratio. OPC and water was mixed in a mixer for $1 \mathrm{~min}$. Then the mixture was placed in the Vicat mould under the rod bearing the needle. The needle was lowered until it comes in contact with the surface of cement paste and the needle was released quickly, allowing it to penetrate into the paste. Repeat this procedure every $2 \mathrm{~min}$ until the needle fails to penetrate the paste about $5 \mathrm{~mm}$ (initial setting) measured from the bottom of the mould and no penetration (final setting). Table 5 shows the results of the initial and final setting of OPC paste with the different dosages of GONF and different mix designs. Both PD and PW shows similar trends in the results. The initial and final settings were decreased with an increasing increment of GONF dosage until $0.05 \%$. This result indicates that the addition of GONF up to $0.05 \%$ in OPC paste mix helps accelerate strength gain within the mix. In contrast, more than $0.05 \%$ of GONF in OPC paste leads to an increase in time of setting. One of the reasons for this behavior may be that a high surface area of GONF results in the reduction of free water which is necessary for cement hydration. The water which was absorbed and held by GONF may affect the speed of cement hydration. The other reason for this increment of setting time can be H-bonds connection between water and GONF. It could affect the speed of cement hydration process.

\subsection{Effect of GONF on the Strength of Hardened Cement Paste and Concrete}

The effect of different percentages of GONF and different mix design methods (Dry- and Wet-mix design methods) on the strength of hardened cement paste was investigated. Figure 7 shows the compressive and flexural strengths of cement paste with different percentages of GONF. The specimen IDs with PD indicate that the specimens were made with Dry-mix design. The results show that the compressive and flexural strengths of PD specimens are higher than the control OPC paste at 7 and 28 days. It clearly shows that the addition of GONF in cement paste mix can lead to an improvement in strength. It is interesting to note that the strength of PD specimens increased with the increasing increment of GONF up to $0.05 \mathrm{wt}$. \% The compressive strength of the PD specimens at 7 and 28 days with $0.05 \%$ GONF increased by $14.9 \%$ and $19.6 \%$, respectively. The flexural strength of PD specimens at 7 and 28 days increased even more when $0.05 \%$ GONF is incorporated $(33.9 \%$ and $28.0 \%$ increments for PD0.05). This phenomenon might be explained with the following rationale. First, GONF as a two-dimensional nanomaterial can interlock with other hydration products thus it prevents nanoand micro-cracks with its own properties (high tensile strength and modulus). Second, the dissolved ions from cement grains may react with oxygen groups at the edge of GONF to produce more cement hydration products such as calcium silicate hydrate (C-S-H) and calcium hydroxide $\left(\mathrm{Ca}(\mathrm{OH})_{2}\right)$ when compared to the control OPC paste. Third, GONF as a nano-scale material can fill the nanoand micro-pores of the cement matrix. This phenomenon is called either nano- or micro-filler effect. 
The optimum content of GONFs for cement paste $(0.05 \%)$ might be due to more cement hydration products and two-dimensional interlocking between hydration products even though there was the reduction of workability (Table 5). In other words, the optimum content of GONFs for cement paste $(0.05 \%)$ was the outcome of offsetting between positive and negative effects of GONFs in cement paste.

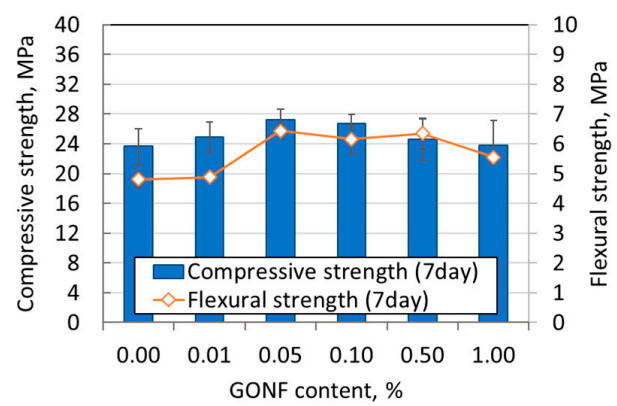

(a)

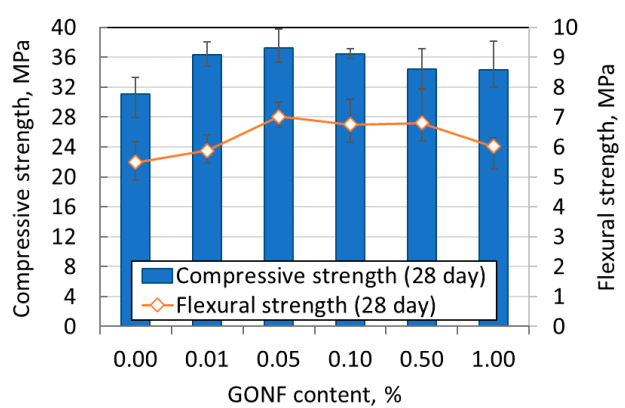

(b)

Figure 7. Compressive and flexural strength results of GONF-combined paste with Dry-mix design method. (a) Mechanical properties of GONF-combined paste with Dry-mix design (PD) (7 days); (b) Mechanical properties of PD (28 days).

To compare the effect of different mix design methods (Dry- and Wet-mix designs), PW specimens which were made with Wet-mix design were tested. The results show the same pattern of strength improvement as compared with PD specimens. All PW specimens have higher compressive and flexural strengths when compared to the control OPC paste (see Figure 8). It also shows that the strength of GONF-combined paste increases with the increasing increment of GONF up to $0.05 \%$. The compressive strength of PW specimen at 7 and 28 days with $0.05 \%$ GONF increase by $26.9 \%$ and $25.5 \%$, respectively. The flexural strength of PW specimens at 7 and 28 days also increased with $0.05 \%$ GONF (34.8\% and 37.3\% increments for PW0.05). As it can be seen, the strengths of PW specimens are higher than the strengths of PD specimens with 0.01 and $0.05 \%$ GONFs. Compressive and flexural strengths at 28 days of PW0.05 are approximately 9\% and 10\% higher than PD0.05. This phenomenon could be explained with the following rationale. First, GONF particles were well dispersed in PW specimens due to the sonication. The sonication can disperse GONF into water well thus there is high possibility that GONF particles can be dispersed well in the cement paste mix. Second, GONF can form H-bond connection with water thus it could affect the fluidity of the cement paste mix. From the results of mini-slump tests, the Wet-mix design method produces bigger areas of slump than the Dry-mix design method. This indicates that the Wet-mix design method may reduce the workability of the cement paste mix below than of the Dry-mix design method. It is known that reduction of workability can affect the strength of the cement composite.

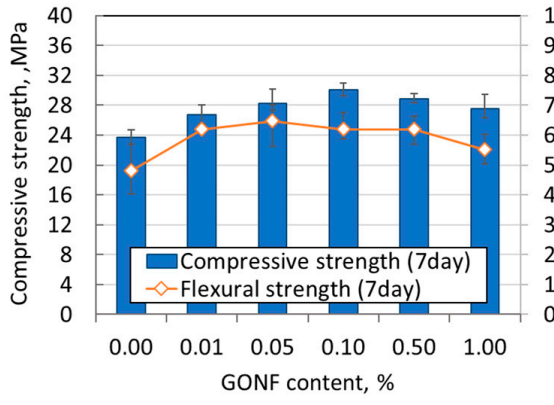

(a)

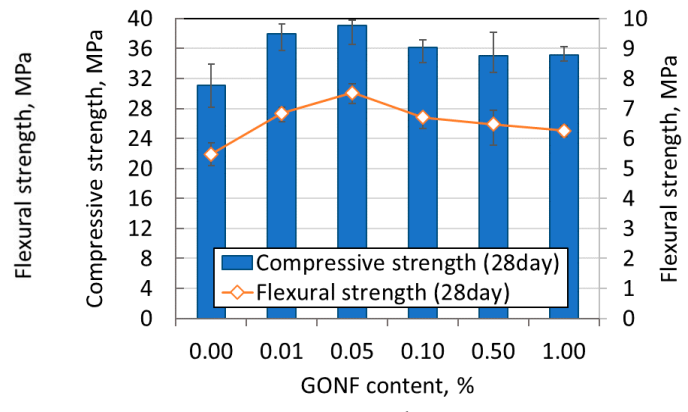

(b)

Figure 8. Compressive and flexural strength results of GONF-combined paste with Wet-mix design method. (a) Mechanical properties of PW (7 days); (b) Mechanical properties of PW (28 days). 
However, even though the ratio of strength increment with PW specimen (Wet-mix design) is higher than PD specimen (Dry-mix design), note that Dry-mix design can still be considered a very practical mix design for GONF-combined cement composites based the results of strength tests.

To investigate the feasibility of GONF use in the concrete industry with no regard for the sonication, dry GONF powder was incorporated into concrete mix. The effect of GONF on the strength of hardened concrete was investigated. The specimen IDs with CD indicate that GONF-combined concrete specimens were made with Dry-mix design. Figure 9 shows the results of compressive and flexural strength tests of $C D$ specimens. As expected, the results indicate that the compressive and flexural strengths of CD specimens are higher than the control Portland cement concrete (PCC) specimens at 7 and 28 days. This result confirms that the addition of GONF in cement composite can lead to the improvement of strength. Another important point to note about the increment of strength is that $0.01 \%$ GONF addition results in $14.2 \%$ strength increment compared to the control PCC specimen. From the strength test results of GONF-combined paste, $0.05 \%$ GONF addition was found as the optimum GONF content. And, this optimum GONF content was the reason to choose the range of GONF content $(0.01-0.1 \%)$ for PCC specimen. For GONF-combined concrete, however, $0.01 \mathrm{wt}$ \% of GONF led to the highest strength increment of CD specimen. The phenomenon that the different optimum contents of GONFs for cement paste $(0.05 \%)$ and concrete $(0.01 \%)$ might be explained with the interaction between cement paste, GONF and aggregates in concrete. As previously mentioned in Section 4.3 , the optimum content of GONFs for cement paste $(0.05 \%)$ might be due to more cement hydration products and two-dimensional interlocking between hydration products even though there was the reduction of workability (Table 5). In other words, the optimum content of GONFs for cement paste $(0.05 \%)$ was the outcome of offsetting between positive and negative effects of GONFs in cement paste. However, for GONF-mixed concrete, the present of dry-aggregates might amplify the reduction of workability even though water correction factor was applied to the concrete mix. Thus, the lowest GONF $(0.01 \%)$ resulted in the highest strength even though there were positive effects of GONF in concrete mix.

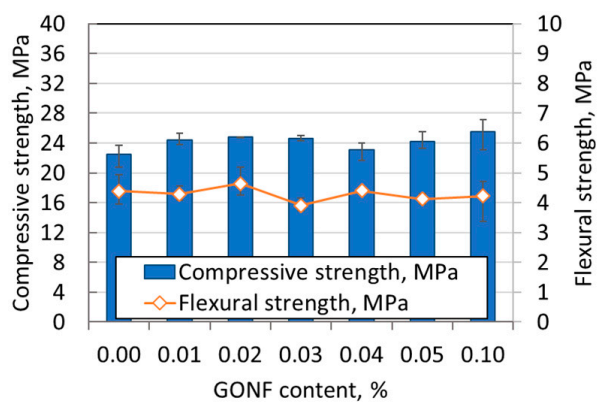

(a)

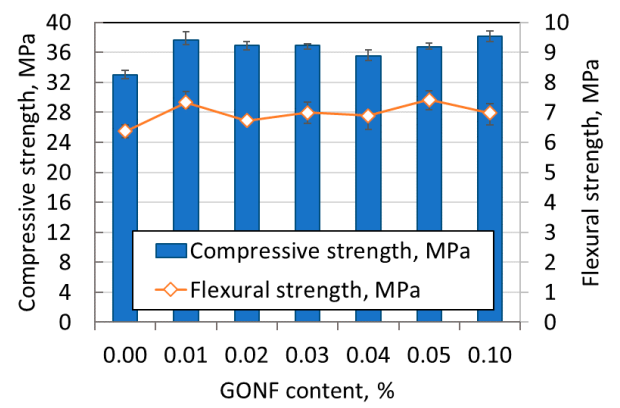

(b)

Figure 9. Compressive and flexural strength results of GONF-combined concrete with Dry-mix design. (a) Mechanical properties of CD (7 days); (b) Mechanical properties of CD (28 days).

\subsection{Characterization of Microstructure of GONF-Composite}

SEM and EDS were employed to investigate microstructure and chemical composition of cement hydration products when GONF is introduced into the cement matrix. The GONF-combined cement paste specimen with $0.05 \%$ GONF was particularly chosen for the characterization of microstructure since $0.05 \%$ GONF was found as an optimal content for strength improvement. Figure 10 shows the overall surface morphology of a GONF-cement paste specimen with $0.05 \%$ of GONF. To investigate the crystalline structure, the magnification was increased from $5000 \mathrm{X}$ (Figure 10a) through $10 \mathrm{kX}$ (Figure 10b). The yellow box indicates the enlarged area under greater magnification. In other words, the yellow box in Figure 10a is the image shown in Figure 10b. The image with the magnification of $5000 \mathrm{X}$ (Figure 10a) reveals several needle like ettringite, platy like crystals and 
early age morphologies of amorphous C-S-H. Figure 10b reveals the structures of the grains and crystals with higher magnification. Needle-shaped ettringite can be clearly seen from Figure 10b protruding into the pores. Amorphous morphology of C-S-H is clearly evident from Figure 10b.

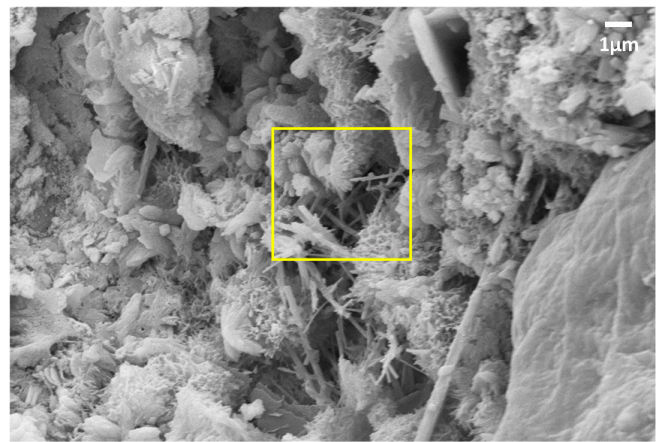

(a)

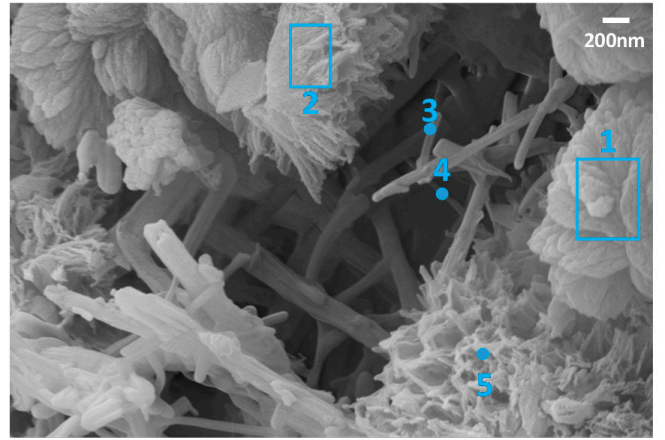

(b)

Figure 10. The images of SEM analysis (GONF-combined cement paste with $0.05 \%$ GONF at 7 days). (a) SEM image with $5000 \mathrm{X}$ magnification; (b) SEM image with $10 \mathrm{kX}$ magnification.

To verify the locations of GONF by detecting carbon element and their contribution to the cement hydration products (e.g., C-S-H), EDS was performed at different spots while SEM images were taken. Each number in Figure 10b indicating either the spot or area shot by the emitted electron beam from EDS to provide the localized elemental information. EDS data is presented in Table 6. As shown in Table 6, the results of two area shots (area shots 1 and 2) and one point shot (point shot of 5) show the presence of carbon and high percentage of oxygen in the hydration products. The data of all area shots show the presence of high amounts of carbon and oxygen including silicon and calcium. Moreover, these two areas which include carbon and high oxygen contents indicate either amorphous C-S-H or crystalline C-S-H which is Jennite. It is well known that C-S-H is the major hydration product which is primarily responsible for the strength in cement paste. On the other hand, the other two point shots (point shots 3 and 4) show that ettringite which is one of byproducts of cement hydration does not have a carbon element. This EDS result is meaningful because most of the GONF is found in C-S-H which is responsible for strength. This observation may draw a conclusion that GONF which is composed of more than 99\% carbon may either act as an initiating point for C-S-H or reinforce C-S-H with its own properties (high tensile strength and modulus).

Table 6. Energy dispersive spectroscopy (EDS) analysis on GONF-cement composite (from SEM image of Figure 10b).

\begin{tabular}{ccccccccccc}
\hline Shots & $\mathbf{C ~ ( \% )}$ & $\mathbf{O}(\%)$ & $\mathbf{A l}(\mathbf{\%})$ & Si (\%) & S (\%) & Ca (\%) & Pd (\%) & Au (\%) & Total (\%) & Probable Compounds \\
\hline 1(Area) & 12.43 & 30.05 & - & 3.17 & - & 50.93 & - & 3.42 & C-S-H + GO \\
2(Area) & 15.56 & 39.99 & 0.86 & 10.15 & - & 25.74 & 4.56 & 3.15 & Jennite + GO \\
3(Point) & - & 13.70 & 2.58 & 9.23 & 5.47 & 69.01 & - & - & 100 & Ettringite \\
4(Point) & - & 7.29 & 1.58 & 12.91 & 2.35 & 75.87 & - & - & Ettringite \\
5(Point) & 16.94 & 39.34 & - & 8.38 & - & 26.00 & 5.62 & 3.72 & C-S-H + GO \\
\hline
\end{tabular}

\section{Conclusions}

This study was aimed at evaluating the feasibility of GONF as an addition in GONF-combined cement composites. To identify the optimum content of GONF to increase the mechanical properties of GONF-combined paste and concrete, different percentages of GONF were added in cement mix. To investigate the effect of mix designs, two different mix design methods were employed (Dry- and Wet-mix design methods). In addition, to verify the practicality of Dry-mix design for GONF-combined concrete, PCC specimens which contain different percentages of dry GONF powder were made, 
cured and tested. A series of laboratory tests were conducted to evaluate mechanical and chemical properties of GONF-combined PCC specimens. The following conclusions have been made:

- It was found that mechanical properties of all GONF-combined paste and concrete have been improved compared to the control specimens. According to compressive and flexural strength tests, highest strengths are observed at GONF of $0.05 \%$ with GON-combined paste and at GONF of $0.01 \%$ with GONF-combined concrete; thus, we conclude that $0.05 \%$ and $0.01 \%$ of GONF are an optimum content in cement paste and concrete.

- Wet-mix designs exhibit higher compressive and flexural strengths than Dry-mix designs. Well-dispersion of GONF in cement paste may contribute the higher strengths. However, Dry-mix design where the GONF is mixed with cement as dry power can be also competitive from a practical viewpoint.

- Petrographic analysis (SEM/EDS) identifies GONFs inside products of cement hydration (surrounded by C-S-H), confirming GONF's a potential role as nano-reinforcing and nano-filling effects.

- To actualize applying this new nanomaterial in concrete industry, Dry-mix design method can be an alternative mix design method.

Acknowledgments: This work is supported by Korea Agency for Infrastructure Technology Advancement grant funded by Ministry of Land, Infrastructure and Transport (Project Number: 17CTAP-C129720-01).

Author Contributions: Boo Hyun Nam and Wonseok Chung conceived the whole procedure of this study. Jinwoo An and Matthew McInnis designed, performed and analyzed the mechanical and petrographic tests.

Conflicts of Interest: The authors declare no conflict of interest.

\section{References}

1. Dreyer, D.R.; Park, S.; Bielawski, C.W.; Ruoff, R.S. The chemistry of graphene oxide. Chem. Soc. Rev. 2010, 39, 228-240. [CrossRef] [PubMed]

2. Zhao, L.; Guo, X.; Liu, Y.; Ge, C.; Guo, L.; Shu, X.; Liu, J. Synergistic effects of silica nanoparticles/polycarboxylate superplasticizer modified graphene oxide on mechanical behavior and hydration process of cement composites. RSC Adv. 2017, 7, 16688-16702. [CrossRef]

3. Pan, Z.; He, L.; Qiu, L.; Korayem, A.H.; Li, G.; Zhu, J.W.; Collins, F.; Li, D.; Duan, W.H.; Wang, M.C. Mechanical properties and microstructure of a graphene oxide-cement composite. Cem. Concr. Compos. 2015, 58, 140-147. [CrossRef]

4. Musso, S.; Tulliani, J.-M.; Ferro, G.; Tagliaferro, A. Influence of carbon nanotubes structure on the mechanical behavior of cement composites. Compos. Sci. Technol. 2009, 69, 1985-1990. [CrossRef]

5. Mohammed, A.; Sanjayan, J.G.; Duan, W.H.; Nazari, A. Incorporating graphene oxide in cement composites: A study of transport properties. Constr. Build. Mater. 2015, 84, 341-347. [CrossRef]

6. Lu, C.; Lu, Z.; Li, Z.; Leung, C.K.Y. Effect of graphene oxide on the mechanical behavior of strain hardening cementitious composites. Constr. Build. Mater. 2016, 120, 457-464. [CrossRef]

7. Li, W.; Li, X.; Chen, S.J.; Long, G.; Liu, Y.M.; Duan, W.H. Effects of nanoalumina and graphene oxide on early-age hydration and mechanical properties of cement paste. J. Mater. Civ. Eng. 2017, 29. [CrossRef]

8. Li, W.; Li, X.; Chen, S.J.; Liu, Y.M.; Duan, W.H.; Shah, S.P. Effects of graphene oxide on early-age hydration and electrical resistivity of portland cement paste. Constr. Build. Mater. 2017, 136, 506-514. [CrossRef]

9. Lv, S.; Ma, Y.; Qiu, C.; Sun, T.; Liu, J.; Zhou, Q. Effect of graphene oxide nanosheets of microstructure and mechanical properties of cement composites. Constr. Build. Mater. 2013, 49, 121-127. [CrossRef]

10. Al Muhit, B.A. Investigation on the mechanical, microstructural, and electrical properties of graphene oxide-cement composite. In Electronic Theses and Dissertations; University of Central Florida: Orlando, FL, USA, 2015.

11. Chuah, S.; Pan, Z.; Sanjayan, J.G.; Wang, C.M.; Duan, W.H. Nano reinforced cement and concrete composites and new perspective from graphene oxide. Constr. Build. Mater. 2014, 73, 113-124. [CrossRef]

12. Gong, K.; Pan, Z.; Korayem, A.H.; Qiu, L.; Li, D.; Collins, F.; Wang, C.M.; Duan, W.H. Reinforcing effects of graphene oxide on portland cement paste. J. Mater. Civ. Eng. 2015, 27. [CrossRef] 
13. Tong, T.; Fan, Z.; Liu, Q.; Wang, S.; Tan, S.; Yu, Q. Investigation of the effects of graphene and graphene oxide nanoplatelets on the micro- and macro-properties of cementitious materials. Constr. Build. Mater. 2016, 106, 102-114. [CrossRef]

14. Sharma, S.; Kothiyal, N.C. Comparative effects of pristine and ball-milled graphene oxide on physico-chemical characteristics of cement mortar nanocomposites. Constr. Build. Mater. 2016, 115, 256-268. [CrossRef]

15. Kiew, S.F.; Kiew, L.V.; Lee, H.B.; Imae, T.; Chung, L.Y. Assessing biocompatibility of graphene oxide-based nanocarriers: A review. J. Control. Release 2016, 226, 217-228. [CrossRef] [PubMed]

16. Garmor has Developed A Simple yet Effective Method of Producing Graphene Oxide. Available online: http:/ / garmortech.com/technology.htm (accessed on 9 July 2015).

17. Blair, R.G.; Chagoya, K.; Biltek, S.; Jackson, S.; Sinclair, A.; Taraboletti, A.; Restrepo, D.T. The scalability in the mechanochemical syntheses of edge functionalized graphene materials and biomass-derived chemicals. Faraday Discuss. 2014, 170, 223-233. [CrossRef] [PubMed]

18. Li, D.; Müller, M.B.; Gilje, S.; Kaner, R.B.; Wallace, G.G. Processable aqueous dispersions of graphene nanosheets. Nat. Nanotechnol. 2008, 3, 101-105. [CrossRef] [PubMed]

19. D'Alessandro, A.; Rallini, M.; Ubertini, F.; Materazzi, A.L.; Kenny, J.M. Investigations on scalable fabrication procedures for self-sensing carbon nanotube cement-matrix composites for shm applications. Cem. Concr. Compos. 2016, 65, 200-213. [CrossRef]

20. Vaisman, L.; Wagner, H.D.; Marom, G. The role of surfactants in dispersion of carbon nanotubes. Adv. Colloid Interface Sci. 2006, 128-130, 37-46. [CrossRef] [PubMed]

21. ASTM. ASTM c150/c150m-17 Standard Specification for Portland Cement; American Society for Testing and Materials: West Conshohocken, PA, USA, 2017.

22. ASTM. ASTM c33/c33m-11a Standard Specification for Concrete Aggregates; American Society for Testing and Materials: West Conshohocken, PA, USA, 2011.

23. ASTM. Standard Test Method for Sieve Analysis of Fine and Coarse Aggregates; American Society for Testing and Materials: West Conshohocken, PA, USA, 2014.

24. Kantro, D.L. Influence of water-reducing admixtures on properties of cement paste-A miniature slump test. Cem. Concr. Aggreg. 1980, 2, 95-102.

25. ASTM. ASTM c191-13 Standard Test Methods for Time of Setting of Hydraulic Cement by Vicat Needle; ASTM International: West Conshohocken, PA, USA, 2013.

26. ASTM. ASTM c127-15 Standard Test Method for Relative Density (Specific Gravity) and Absorption of Coarse Aggregate; American Society for Testing and Materials: West Conshohocken, PA, USA, 2015.

27. ASTM. ASTM c128-15 Standard Test Method for Relative Density (Specific Gravity) and Absorption of Fine Aggregate; American Society for Testing and Materials: West Conshohocken, PA, USA, 2015.

28. Camilli, L.; Pisani, C.; Gautron, E.; Scarselli, M.; Castrucci, P.; D’Orazio, F.; Passacantando, M.; Moscone, D.; Crescenzi, M.D. A three-dimensional carbon nanotube network for water treatment. Nanotechnology 2014, 25, 065701. [CrossRef] [PubMed]

29. Saner, B.; Gürsel, S.A.; YÜRÜM, Y. Layer-by-layer polypyrrole coated graphite oxide and graphene nanosheets as catalyst support materials for fuel cells. Fuller. Nanotub. Carbon Nanostruct. 2013, 21, 233-247. [CrossRef]

30. Wu, H.; Chan, G.; Choi, J.W.; Ryu, I.; Yao, Y.; McDowell, M.T.; Lee, S.W.; Jackson, A.; Yang, Y.; Hu, L.; et al. Stable cycling of double-walled silicon nanotube battery anodes through solid-electrolyte interphase control. Nat. Nanotechnol. 2012, 7, 310-315. [CrossRef] [PubMed]

(C) 2018 by the authors. Licensee MDPI, Basel, Switzerland. This article is an open access article distributed under the terms and conditions of the Creative Commons Attribution (CC BY) license (http:/ / creativecommons.org/licenses/by/4.0/). 